\title{
Tuberculosis burden in China: a high prevalence of pulmonary tuberculosis in household contacts with and without symptoms
}

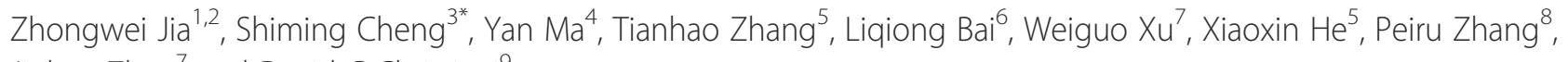
Jinkou Zhao ${ }^{7}$ and David C Christiani ${ }^{9}$

\begin{abstract}
Background: In the context of decreasing tuberculosis prevalence in China, we examined the effectiveness of screening household contacts of tuberculosis patients.

Methods: A tuberculosis survey was conducted in 2008. All 3,355 household contacts of notified tuberculosis cases were examined with a questionnaire interview, chest $X$-ray and three sputum smear tests. The effectiveness was examined by comparing the prevalence of pulmonary tuberculosis in household contacts with or without presenting clinical symptoms against the respective notification rates. Regression models were used to evaluate the factors associated with pulmonary tuberculosis.

Results: Of the 3,355 household contacts, 92 members (2.7\%) had pulmonary tuberculosis, among which 46 cases were asymptomatic. The prevalence of pulmonary tuberculosis and smear positive cases in household contacts without symptoms were 20 and 7 times higher than the notification rates in 2008, while those in household contacts with symptoms were 247 and 108 times higher than notification rates, respectively. The patients detected were mainly Index Cases' spouses, sisters/brothers and those who were in contact with female Index Cases.

Conclusions: The present study provides convincing evidence that household contacts of notified tuberculosis cases are at higher risk of developing tuberculosis. Routine screening for household contacts without any symptoms is recommended for sustained tuberculosis control in China as well as in the world.
\end{abstract}

Keywords: Tuberculosis, Household contact, Index cases

\section{Background}

A few studies have indicated that close contacts have a higher risk of developing active tuberculosis [1-5]. China national tuberculosis program has yet included case detection among this group due to inadequate resources in the past. By 2005, the coverage of DOTS has achieved $100 \%$ at county scale while the case detection rate and the cure rate of the new sputum smear positive patients reached $79 \%$ and $91 \%$ respectively in China, indicating that China met the WHO's staggered global targets for detection and treatment of tuberculosis cases [6-9].

\footnotetext{
* Correspondence: smcheng@chinatb.org

${ }^{3}$ National Center for Tuberculosis Control and Prevention, Chinese Center for Disease Control and Prevention, Beijing 100017, China

Full list of author information is available at the end of the article
}

Given the nature of tuberculosis surveillance being passive, most patients could not be detected until they present symptoms and seek health care [10]. In 2007, China's Ministry of Health piloted a new screening strategy among suspects in household contacts of notified tuberculosis cases (Index Cases) in several regions to inform a possible screening strategy nationwide [11]. More positive pulmonary tuberculosis patients have been detected in this pilot than routine surveillance [2], which inspired a more extensive case detection among all household contacts considering the Mega health investment in health medical reform to eliminate tuberculosis from population [12]. Since 2008, a rigid screening strategy in household contacts with symptoms has been tentatively extended to the household contacts without symptoms.

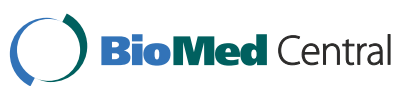


The present study examines effectiveness of the screening strategies among the household contacts with or without symptoms, to inform whether the screening strategy should cover the household contacts without symptoms.

\section{Methods}

For the present study, the Index Case is defined as a smear positive pulmonary patient who was first to be diagnosed in the household, but not always the first real patient. To our knowledge, there is no consistent definition of household contacts [13]. In our study, the household contacts are either family members or notfamily members who have been living in same house with the Index Case for more than two weeks in the past three months before the data when the Index Cases were diagnosed. Notified cases were the number of pulmonary tuberculosis cases registered in tuberculosis national surveillance system (TBNSS). We calculated notification rate by dividing the number of notified cases by the number of total population in a geographically defined location. The prevalence among household contacts was calculated as the percentage of pulmonary tuberculosis cases among 100,000 household contacts. Delayed reporting was defined as the interval being more than two weeks between the onset of any symptom and the presentation to a health care provider [14].

\section{Study settings and cases}

A cross-sectional survey was conducted among household contacts following the detection of the Index Case in the household in 2008. Based on the levels of tuberculosis burden in China [7], four regions, namely Eastern, Western, Central and Metropolitan regions were selected for the present study [7]. Two counties or urban districts in each region were selected, covering about 8.8 million populations (Figure 1). Since April, 1 2008, immediately after each of the Index Cases were diagnosed and notified by the local health professionals, all family members or relatives who have been living in same house with the Index Case for more than two weeks in the past three months before the data when the Index Cases were diagnosed (household contacts) were referred for a screening. All household contacts were administered a questionnaire interview by local health professionals, a chest X-ray examination at the local health facility, three sputum smear tests (the previous evening, the morning and at the moment of the day when seeing the local health professionals) were carried out at the county reference laboratories.

The demographic data, such as age, sex, have been gathered for both Index Cases and household contacts. Additional information were collected for the Index Cases, including education, whether or not re-treated cases, whether or not cavitary pulmonary tuberculosis, the onset of symptoms and the date of the first visit to local health facility. Additional information were also collected for household contacts, including whether having any symptoms, whether living in the same bedroom and the relationships with the Index Cases.

The primary outcome measurement is the prevalence pulmonary tuberculosis among the household contacts

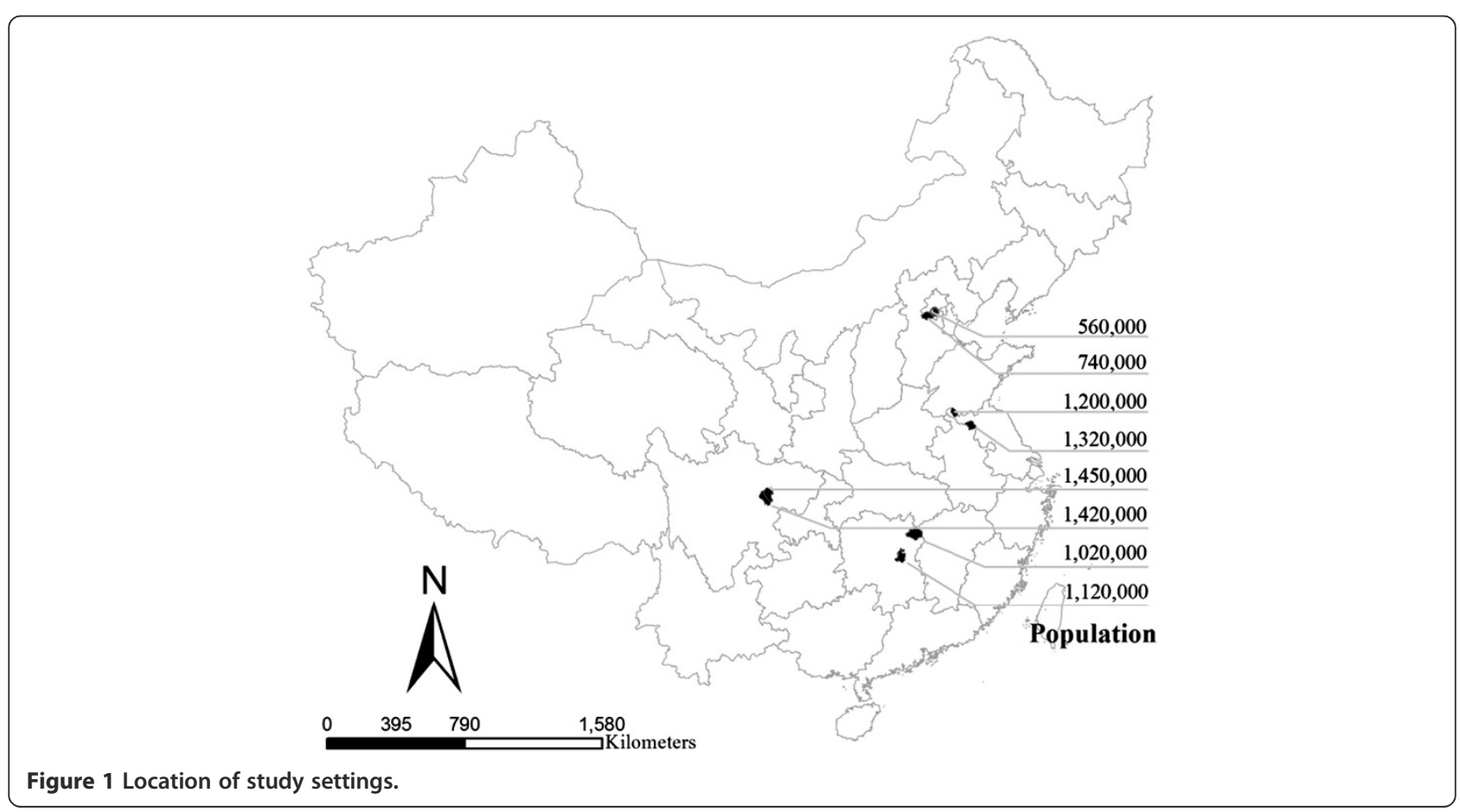


by whether having any symptoms while the secondary measurement is the effectiveness of the screening strategy among contacts without presenting any symptoms. All tuberculosis cases were diagnosed according to the latest guidelines issued by China Ministry of Health in 2003 [15]. For a diagnosis of smear-positive tuberculosis, one of three sets of conditions should be met: (1) 2 positive sputum smears by microscopy, (2) 1 positive sputum smear and 1 positive sputum culture, or (3) 1positive sputum smear-positive with typical pathology of active tuberculosis on a chest X-ray [15]. For a diagnosis of sputum smear-negative TB, the expert groups based on results of chest X-ray, purified protein derivative (PPD) and clinical manifestations, one of four conditions should be met after three negative sputum smear and 1 negative sputum culture: (1) typical pathology of active tuberculosis on a chest X-ray following symptoms such as cough, haemoptysis and fever, (2) typical pathology of active tuberculosis on a chest X-ray and diameter of PPD $>20 \mathrm{~mm}$, (3) typical pathology of active tuberculosis on a chest X-ray and pathological changes of tuberculosis in extra-pulmonary tissues, (4) excluding other pulmonary diseases after following up or anti TB treatment for suspicious cases for three weeks. Active pulmonary tuberculosis cases include either smear positive or negative pulmonary tuberculosis cases [15].

\section{Ethical issues}

This study was approved by the Chinese Ethical Committee for Tuberculosis Operational Research, Chinese Center for Disease Control and Prevention. A written informed consent was obtained from each of all participants prior to the interview.

\section{Statistical analysis}

We compare the prevalence of pulmonary tuberculosis in household contacts who have presented symptoms to those who have not yet presented symptoms to examine the difference. We also compare the prevalence of pulmonary tuberculosis in the contacts with or without symptoms to the notification rate.

The factors associated with the transmission of pulmonary tuberculosis were examined by using a multilevel logistic saturated regression with the clinical diagnosis of pulmonary tuberculosis as an outcome. Independent variables included three kinds of variables. The first kind was characteristics of the index patients: age (0-14, 15-64 and $>65)$, sex (0: women, 1 : men), cavitary disease (yes:1, no: 0 ), delay (yes:1, no :0) and treatment history (new: 0 , retreatment: 1 ). The second kind was characteristics of the household contacts: age (0-14, 15-64 and >65), sex (0: women, 1: men) and symptoms (yes: 1, no: 0 ). The third kind was links between index patients and their contacts: relationship (spouse, parents, children, sister/brother and other), whether living same bedroom for index patients (yes: 1, no: 0 ). In the model, the household was fitted as 2-level and each contact as 1 level. Relative risks were calculated as crude odds ratios and adjusted odds ratios with $95 \%$ confidence intervals (CI) and a P value below0.05 indicates a statistical significance. All data were double entered using EpiData and were analyzedusingMLwiN2.02 and SPSS 17.

\section{Results}

Out of 1,575 households of Index Cases, 3,381 household contacts were identified and 3,355 (99.2\%) examined (Figure 2). Most Index Cases $(1,412 / 1,575)$ were the new positive cases, $77 \%$ were men and $80 \%$ were reported more than two weeks (delayed reporting) (Table 1).

Of the 3,355 household contacts, 92 people had pulmonary tuberculosis (2,742 per 100,000 populations) with 21 being smear positive cases (626 per 100,000 populations). Of 92 pulmonary tuberculosis diagnosed, 46 cases (9 smear positive cases) had not yet presented any clinical symptoms (Table 1). For household contacts without symptom, the prevalence of pulmonary tuberculosis (1,483 per 100,000 populations) was 20 times higher than the notified rate (73 per 100,000 populations) and the prevalence of smear positive cases (290 per 100,000 populations) was 7 times higher than the notification rate (43 per 100,000 populations). Among household contacts with symptoms, the prevalence of pulmonary tuberculosis and smear positive cases were 248 (18,110 per 100,000 populations) and 107 (4,724 per 100,000 populations) times higher than the notification rates respectively (Table 1 ).

By adjusting household clustering, household contacts presenting symptoms had 14 times higher risk to be diagnosed as pulmonary tuberculosis in comparison with the those who have not yet presented any symptoms (OR13.94, $\mathrm{P}<0.05)$ (Table 2). The pulmonary tuberculosis was more likely to be found among the spouses or sisters/ brothers of the Index Cases and among those who were in contact with the female Index Cases (Table 2). However, the pulmonary tuberculosis was not found associated with age, cavitary lesions, treatment history of the Index Cases and living in the same bedroom with the Index Cases tuberculosis (Table 2). Among household contacts, the elder people (aged above 65 years old) (AOR4.96, $\mathrm{P}<0.05)$ and men (AOR1.52, $\mathrm{P}<0.05)$ were most likely to be diagnosed tuberculosis (Table 2).

\section{Discussion}

The study presents very high prevalence of pulmonary tuberculosis in the household contacts with or without any symptoms, which are about six times or nine times higher in comparison with report of 2010 national tuberculosis prevalence survey (2742 per 100,000 populations 


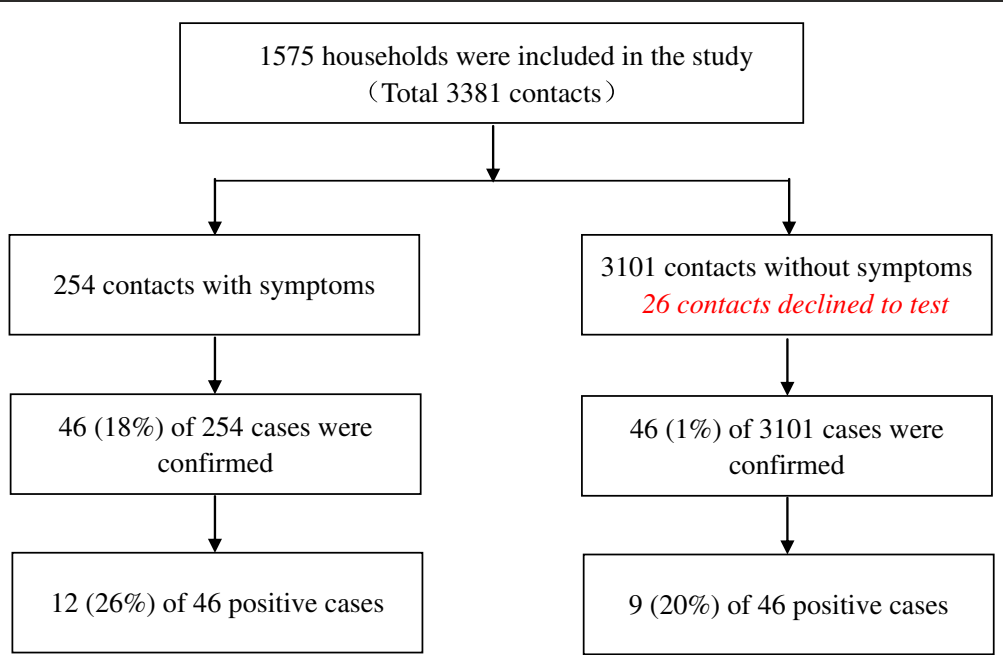

Figure 2 Summary of screening process.

vs. 459 per 100,000 populations and 626 per 100,000 populations vs. 66 per 100,000 populations) respectively [16]. Assuming the difference in prevalence between the present study and 2010 national tuberculosis survey can be extrapolated to nationwide, there might have been 30,748 undiagnosed pulmonary tuberculosis cases nationwide if all the household contacts of all notified cases in 2008 were screened. This presents a great missed opportunity to detect a large pool of pulmonary tuberculosis in the population to further advance the remarkable achievement in past two decades with fighting against tuberculosis in China [9].

Not surprising, our study find that the household contacts presenting symptoms have 14 times the likelihood (OR95\% CI, 13.94 $(8.61,2.59)$ ) to be diagnosed as pulmonary tuberculosis comparing with those have not yet presented any symptoms (Table 2). This finding is consistent with previous studies but we could not determine whether the household contacts have been infected by TB cases inside or outside the family $[1,2]$. However, we find the prevalence of pulmonary tuberculosis and positive tuberculosis are respectively 20 times and 7 times higher than the notification rates which is publicly reported (Tables 1 \& 2). This implies that early active case finding and treatment of tuberculosis patients without presenting symptoms among close contacts might bring benefit for the general public health. Although whether the screening strategy among the household contacts is cost-effective remains controversial, it is obvious that eradicating tuberculosis from the population totally needs to identify all potential transmission sources and eliminate them $[17,18]$. This concept of costeffectiveness should be observed in a longer period of term and from a global perspective rather than measuring the cost and in local areas only. Furthermore, most Index Cases had a history of TB before being found and might contribute to drug resistance which will worsen TB burden. The

Table 1 Occurrence of tuberculosis among the household contacts with and without symptoms by regions in 2008

\begin{tabular}{|c|c|c|c|c|c|c|c|c|c|c|c|}
\hline & \multirow{2}{*}{$\begin{array}{c}\text { Index } \\
\text { patients }\end{array}$} & \multicolumn{3}{|c|}{ Overall } & \multirow[b]{2}{*}{$\begin{array}{c}\text { Notification } \\
1 / 100,000\end{array}$} & \multicolumn{3}{|c|}{ With symptoms } & \multicolumn{3}{|c|}{ Without symptoms } \\
\hline & & Case & Contact & $\begin{array}{c}\text { Prevalence } \\
1 / 100,000\end{array}$ & & Case & Contact & $\begin{array}{c}\text { Prevalence } \\
1 / 100,000\end{array}$ & Case & Contact & $\begin{array}{c}\text { Prevalence } \\
1 / 100,000\end{array}$ \\
\hline Overall & 1575 & 92 & 3355 & 2742 & 73.33 & 46 & 254 & 18110 & 46 & 3101 & 1483 \\
\hline Positive cases & 1575 & 21 & 3355 & 626 & 43.94 & 12 & 254 & 4724 & 9 & 3101 & 290 \\
\hline \multicolumn{12}{|l|}{ By regions } \\
\hline Mega polis & 175 & 2 & 363 & 551 & 21.77 & 1 & 15 & 6667 & 1 & 348 & 287 \\
\hline Centre region & 437 & 26 & 661 & 3933 & 77.22 & 9 & 46 & 19565 & 17 & 615 & 2764 \\
\hline Eastern region & 465 & 58 & 1051 & 5519 & 81.05 & 34 & 150 & 22667 & 24 & 901 & 2664 \\
\hline Western region & 498 & 6 & 1280 & 469 & 79.93 & 2 & 43 & 4651 & 4 & 1237 & 323 \\
\hline
\end{tabular}


Table 2 Factors related with occurrence of tuberculosis in household contacts

\begin{tabular}{|c|c|c|c|c|c|c|}
\hline & $\begin{array}{l}\text { Number } \\
\text { of IC* }\end{array}$ & $\begin{array}{l}\text { Number of } \\
\text { contacts }\end{array}$ & $\begin{array}{l}\text { Cases in } \\
\text { contacts }\end{array}$ & $\begin{array}{l}\text { Prevalence } \\
(1 / 100,000) \\
\end{array}$ & OR & AORt \\
\hline \multicolumn{7}{|l|}{ Index patients } \\
\hline \multicolumn{7}{|l|}{ Age } \\
\hline $0-14$ & 7 & 12 & 1 & 8333 & 1.00 & 1.00 \\
\hline $15-64$ & 1224 & 2568 & 69 & 2687 & $0.31(0.03,3.48)$ & $0.40(0.03,1.50)$ \\
\hline $65-$ & 334 & 773 & 22 & 2846 & $0.33(0.03,3.87)$ & $0.46(0.04,5.40)$ \\
\hline \multicolumn{7}{|l|}{ Sex } \\
\hline Woman & 434 & 967 & 37 & 3826 & 1.00 & 1.00 \\
\hline Man & 1141 & 2388 & 55 & 2303 & $0.59(0.37,0.94) \neq$ & $0.60(0.36,0.99) \neq$ \\
\hline \multicolumn{7}{|l|}{ Cavitary disease } \\
\hline No & 1153 & 2471 & 66 & 2671 & 1.00 & 1.00 \\
\hline Yes & 414 & 868 & 26 & 2995 & $1.12(0.67,1.86)$ & $1.22(0.72,2.07)$ \\
\hline \multicolumn{7}{|l|}{ Treatment history } \\
\hline New & 1427 & 3089 & 80 & 2590 & 1.00 & 1.00 \\
\hline Re-treatment & 144 & 258 & 12 & 4651 & $1.75(0.80,3.38)$ & $1.65(0.45,3.23)$ \\
\hline \multicolumn{7}{|l|}{ Treatment delay } \\
\hline No & 304 & 698 & 11 & 1576 & 1.00 & 1.00 \\
\hline Yes & 1217 & 2657 & 81 & 3049 & $1.91(0.98,3.73)$ & $1.70(0.85,3.71)$ \\
\hline \multicolumn{7}{|c|}{ Household contacts } \\
\hline \multicolumn{7}{|l|}{ Age } \\
\hline $0-14$ & & 359 & 4 & 1114 & 1.00 & 1.00 \\
\hline $15-64$ & & 2649 & 70 & 2643 & $2.28(0.83,6.28)$ & $2.30(0.84,6.30)$ \\
\hline $65-$ & & 342 & 18 & 5263 & $4.77(1.59,14.37) \neq$ & $4.96(1.85,14.89) \neq$ \\
\hline \multicolumn{7}{|l|}{ Sex } \\
\hline Woman & & 1795 & 42 & 2340 & 1.00 & 1.00 \\
\hline Man & & 1560 & 50 & 3205 & $1.40(0.91,2.14) \neq$ & $1.52(1.18,2.32) \neq$ \\
\hline \multicolumn{7}{|l|}{ Symptoms } \\
\hline No & & 3101 & 46 & 1483 & 1.00 & 1.00 \\
\hline Yes & & 254 & 46 & 18110 & $14.10(8.91,22.31) \neq$ & $13.94(8.61,12.59) \neq$ \\
\hline \multicolumn{7}{|c|}{ Index patients and household contacts } \\
\hline \multicolumn{7}{|l|}{ Relationship } \\
\hline Couple & & 949 & 39 & 4110 & 1.00 & 1.00 \\
\hline Parent & & 654 & 20 & 3058 & $0.73(0.41,1.29)$ & $0.55(0.20,1.49)$ \\
\hline Children & & 939 & 18 & 1917 & $0.44(0.24,0.79) \neq$ & $0.35(0.12,0.92) \neq$ \\
\hline Sister/brother & & 242 & 10 & 4132 & $0.86(0.39,1.89)$ & $0.72(0.23,2.28)$ \\
\hline Other & & 544 & 5 & 921 & $0.22(0.08,0.56) \neq$ & $0.40(0.21,0.77) \neq$ \\
\hline \multicolumn{7}{|c|}{ Whether living same bedroom } \\
\hline No & & 2368 & 55 & 2323 & 1.00 & 1.00 \\
\hline Yes & & 987 & 37 & 3749 & $1.70(1.09,2.63) \neq$ & $1.35(0.74,2.50)$ \\
\hline \multicolumn{7}{|l|}{ Random effect } \\
\hline Constant & & & & & & $3.08(1.05,9.09) \neq$ \\
\hline
\end{tabular}

Remark: * IC is Index Cases. TAOR is adjusted odds ratios. $\ddagger$ indicate $95 \%$ significant.

on-going medical reform in China may increase the budget and expenditure on tuberculosis control [12]. Screening all household contacts of Index Cases is feasible and also a key step to bring tuberculosis control to sustainability in China.
In fact, Index Cases are not always detected by passive surveillance now and increased numbers of tuberculosis patients have been detected by improved education and routine physical examinations [19]. 
In contrast to men, women are about 1.7 times more likely to transmit pulmonary tuberculosis to the household contacts while the male household contacts are more easily infected (AOR1.5) (Table 2). This may be due to the fact that women have more chance and longer time to be in contact with other family members. A local study and the national survey in rural areas in 2008 all indicate that about $74 \%$ of rural women's work is responsible for taking care children and elderly people at home $[20,21]$. In the present study, more than $80 \%$ of Index Cases are in the rural areas, consistent with the distribution of tuberculosis in China $[7,16]$.

The data from the present study do not support our hypothesis that cavitary tuberculosis, new smear positive cases and delayed reported patients may be associated with a higher probability of transmission within the home (Table 2). These findings may imply that Index Cases may not be the first patient in the family although they are the cases notified. Another unexpected outcome is the higher rate of non-tuberculosis cases in household contacts with symptoms (34/46 with symptoms vs. 37/ 46 without symptoms), which might be expected to be lower $(<50 \%)$ in view of the low HIV prevalence in China. This unusual result might be due to wide mobilization during the study which may result in overstated symptoms when the household contacts were interviewed. Clearly further study is needed.

No bacterial genotyping was performed the present study. This disables us to identify whether the household contacts are infected by Index Cases or in the community. However, convincing evidence in the present study indicates that household contacts have more exposure to tuberculosis. There are a large number of potential pulmonary tuberculosis cases yet to be detected in China, irrespective of having symptoms or not. We also did not assessed HIV status for both Index Cases and the household contacts, which might influence our results, but China is still low HIV prevalence, which limits HIV/tuberculosis co-infection as a major confounder [22]. The type of building and ventilation of house might be associated with TB infection, but our study focused on rural areas where TB cases account for more than $80 \%$ in China. In these areas, there is little difference in the type of building and ventilation of house, so this factor will is not main contribution to difference in $\mathrm{TB}$ infection.

\section{Conclusion}

Despite the limitation and controversies, the present study highlights that screening only the symptomatic contacts is clearly not enough to sustain the achievement of tuberculosis control in China, as well as in the world.
Summary box-What is already known on this subject and what does this study add to? And policy implications

A few studies indicated that close contacts have a higher risk of developing active tuberculosis. China initiated a screening strategy for household contacts with suspicious symptoms of Index Cases in 2007 and more pulmonary tuberculosis patients have been notified than the routine surveillance. But national tuberculosis program has not included the screening among household contacts without symptoms due to inadequate resources in the past.

The present study examined the effectiveness of screening the household contacts without symptoms in 2008 and compared the effectiveness of screening household contacts with and without symptoms.

The on-going medical reform with mega health investment in China provides the promising opportunity for further improvement of tuberculosis case finding in China, including screening among household contacts without symptoms. The present study provides strong evidence to new tuberculosis screening guideline in China.

\section{Competing interests}

The authors declare that they have no competing interests.

\section{Authors' contribution}

ZJ, SC designed this study, ZJ, SC, TZ and YM analyzed and explained the data, ZJ gave the first draft of the paper and ZJ, JZ and DC wrote final paper, $L B, W X, X H$ and $W Z$ contribute the data collection, all authors read the paper and contribute the work. All authors read and approved the final manuscript.

\section{Acknowledgments}

This work was supported by National Nature Science Foundation (No. 81372958) and Takemi Fellowship Program in Harvard School of Public Health.The funding organization does not have a role in study design, data collection, analysis, decision to publish, or preparation of the manuscript.

\section{Author details}

${ }^{1}$ National Institute of Drug Dependence, Peking University, Beijing 100191 China. ${ }^{2} T a k e m i$ Program in International Health, Department of Global Health and Population, Harvard School of Public Health, Boston, MA, USA. ${ }^{3}$ National Center for Tuberculosis Control and Prevention, Chinese Center for Disease Control and Prevention, Beijing 100017, China. ${ }^{4}$ Center office, Beijing Tuberculosis and Thoracic Tumor Research Institute, Beijing 101149, China. ${ }^{5}$ Beijing Research Institute for Tuberculosis Control, Beijing 100035, China.

${ }^{6}$ Hunan institute for Tuberculosis Control, Changsha 410013, China. ${ }^{7}$ Jiangsu Center for Disease Control and Prevention, Nanjing 210009, China. ${ }^{8}$ Sichuan Center for Disease Control and Prevention, Chengdu 610041, China. ${ }^{9}$ Department of Environment health, Harvard School of Public Health, Boston, MA, USA.

Received: 4 July 2013 Accepted: 27 January 2014 Published: 6 February 2014

\section{References}

1. Tao HB, Chen P, Guo SL, Yu Y: Investigation on the close contact of the rural patients with pulmonary tuberculosis. Chin J Public Health 2008, 24(6):672-673.

2. $\mathrm{Xu} \mathrm{C}, \mathrm{Hu}$ B: Prevalence of active pulmonary tuberculosisamong household contacts of recently diagnosed pulmonary tuberculosispatients with positive sputum-smear. Zhonghua Liu Xing Bing Xue Za Zhi (Chinese) 2008, 29(7):693-695. 
3. Radhakrishna S, Frieden TR, Subramani R, Santha T, Narayanan PR: Additional risk of developing TUBERCULOSIS for household members with a TB case at home at intake: a 15-year study. Int J Tuberc Lung Dis 2007, 11:282.

4. Jackson-Sillah D, Hill PC, Fox A, et al: Screening for tuberculosisamong 2381 household contacts of sputum-smear-positive cases in The Gambia. Trans R Soc Trop Med Hyg 2007, 101:594-601.

5. Noertjojo K, Tam CM, Chan L, et al: Contact examination for tuberculosisin Hong Kong is useful [J]. Int J Tuberc Lung Dis 2002, 6(1):19-24.

6. China Tuberculosis Control Collaboration: The effect of tuberculosis control in China. Lancet 2004, 364:417-22.

7. Jia ZW, Cheng SM, Li ZJ, et al: Combining domestic and foreign investment to expand tuberculosis control in China. PLoS Med 2010 7:e1000371.

8. World Health Organization: Global Tuberculosiscontrol. ; 2011

9. Ministry of Health P.R.China, National Development and Reform Commission and Ministry of Finance P.R. China: Assessment on schemes of the national tuberculosisprevention and control plan (2001-2010). Beijing: Military Medical Science Press; 2012.

10. Shi JS, Zhang HM, Zheng DM, Shi LK: The evolution of detection strategy for tuberculosis (TB)]. Beijing China 1980 2009, 39:226-228.

11. Disease prevention and Control Bureau of the Ministry of health: Guideline of screening for household contacts of the new notified sputum smear cases [Health 12]. ; 2006.

12. Liu P: China to Invest 850 billion yuan in Medical Reform. ; 2009. Avaiable at http://www.eeo.com.cn/ens/2009/0218/129659.shtml. Access at April, 6.

13. Singh J, Sankar MM, Kumar S, et al: Incidence and prevalence of tuberculosis among household contacts of pulmonary tuberculosis patients in a peri-urban population of South Delhi. India PLOS ONE 2013, 8(7):e69730.

14. TB Control Project, Ministry of health: Manual of the tuberculosiscontrol project of the world bank loan in China [M]. 2nded. Beijing: Office of TB Control Project, Ministry of Health; 2007. in Chinese.

15. Bureau of Health Standards, Health Supervision, and Ministry of Health P.R. China: Collection of diagnostic standard and correlative laws and regulations for infectious disease. Beijing, BJ: China Standard Press; 2003.

16. Technical Guidance Group of the Fifth National TB Epidemiological Survey: The Office of the Fifth National TB Epidemiological Survey. The fifth national tuberculosisepidemiological survey in 2010. Chin $\mathrm{J}$ Antituberculosis 2012, 34(8):485-508.

17. Samandari T, Bishai $D$, Luteijn $M$, et al: Costs and consequences of additional chest $\mathrm{x}$-ray in a tuberculosisprevention program in Botswana. Am J Respir Crit Care Med 2011, 183:1103-1111.

18. Chang KC, Leung CC, Tam CM: Household contact investigation of tuberculosis in low-income and middle-income countries: public-health impact. Lancet Infect Dis 2009, 9(1):3-4.

19. Cheng $S$, Chen $W$, Yang $Y$, et al: Effect of diagnostic and treatment delay on the risk of tuberculosis transmission in Shenzhen, China: an observational cohort study, 1993-2010. PLoS One 2013, 8(6):e67516.

20. Guo JX: Stand at a nonplus of the rural women in China. Beijing Cultural Rev 2011, 2:92-97.

21. Zhen S: National survey on status of women in rural areas. Social Sciences Academic Press; 2008:2. Access at Jan 30, 2014 at http://www.xingbie.org/ newsdetail.asp?id=1486.

22. Jia Z, Wang L, Chen RY, et al: Tracking the evolution of HIV/AIDS in China from 1989-2009 to inform future prevention and control efforts. PLOS One 2011, 6(10):e25671.

doi:10.1186/1471-2334-14-64

Cite this article as: Jia et al:: Tuberculosis burden in China: a high prevalence of pulmonary tuberculosis in household contacts with and without symptoms. BMC Infectious Diseases 2014 14:64.

\section{Submit your next manuscript to BioMed Central and take full advantage of:}

- Convenient online submission

- Thorough peer review

- No space constraints or color figure charges

- Immediate publication on acceptance

- Inclusion in PubMed, CAS, Scopus and Google Scholar

- Research which is freely available for redistribution

Submit your manuscript at www.biomedcentral.com/submit
C Biomed Central 